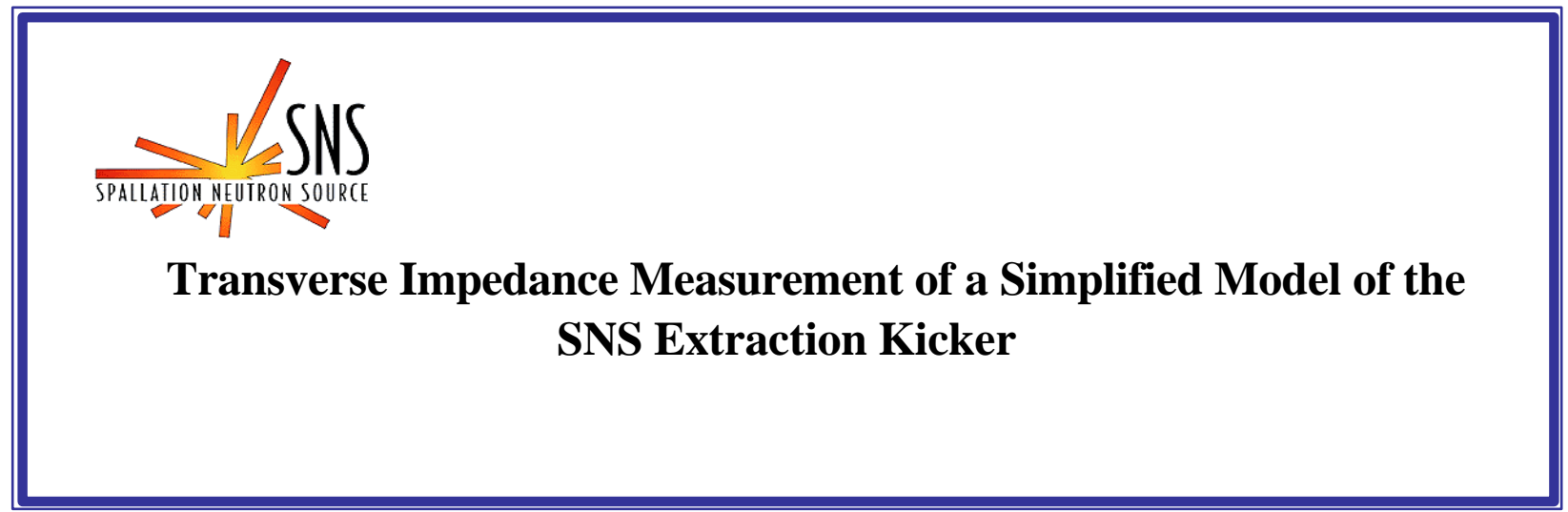

BNL/SNS TECHNICAL NOTE

NO. 088

D. Davino and H. Hahn

March, 2001

COLLIDER-ACCELERATOR DEPARTMENT

BROOKHAVEN NATIONAL LABORATORY

UPTON, NEW YORK 11973 


\title{
Transverse Impedance Measurement of a Simplified Model of the SNS Extraction Kicker
}

\author{
D. Davino and H. Hahn \\ Brookhaven National Laboratory \\ Upton, NY 11973 USA
}

March, 2001

\begin{abstract}
In this technical report we present some measurements and comments on the transverse coupling impedance of a simplified model for the SNS accumulator ring extraction kicker.
\end{abstract}

\section{Introduction}

The SNS Accumulator Ring high intensity beam needs an accurate estimate of the longitudinal and transverse coupling impedance for all the ring components [1]. This estimate is usually called impedance budget. Previous studies and measurements have pointed to the extraction kicker as the major contributor to the impedance budget [2], so that intensive studies have been carried out to find a solution to reduce that contribution.

In the ASAC Review of September 2000, Y.Y. Lee has proposed a new type of winding for the kicker ferrite which is supposed to reduce the transverse impedance without degrading the other performances of the extraction kicker, namely the kicking magnetic field and its rise time [3]. In this report we refer to that winding with the name YY Loop.

Another possibility to reduce the transverse impedance is to act on the PFN termination, since previous studies [4] have been shown that a reduction of the pulser impedance seen through the PFN port gives a reduction of the transverse impedance too.

This technical report gives a preliminary answer on

- The effect of the winding (YY Loop) on the transverse impedance.

- The effect of the PFN termination on the transverse impedance. 
We use the word preliminary because we proceed on a simplified model of the extraction kicker with different and shorter dimensions. This fact has some implication, the most important of which is that, with the design dimensions, the kicker transverse impedance will have a resonance behavior around $50 \mathrm{MHz}$, then in a range of frequencies which could be critical for instability issue. Instead, in the present case the lower resonance is around $180 \mathrm{MHz}$, then out of the range of frequencies under observation.

Finally, the idea of this report is to give, at least, the trends of the quantities under observation. However, it is planned to measure a real prototype of the extraction kicker in May 2001.

\section{The Scheme of Measurement}

We used the two wires standard technique [5, 6]. All the measurement were made using a Network Analyzer Agilent 8753ES using 1601 points, in a logarithmical scale, from $30 \mathrm{kHz}$ to $100 \mathrm{MHz}$. We measured the transmission coefficient $S_{21}$ through a double wire cable, perpendicularly disposed with respect to the kicker plates. Two wide-band transformers were used to match the $400 \Omega$ characteristic impedance of the cable to the $50 \Omega$ impedance of the instrument cables. The range of reliable frequencies for the transformers are $30 \mathrm{kHz}$ to $100 \mathrm{MHz}$. Then, we used the so-called log-formula:

$$
Z_{t r}=\frac{c}{\omega \Delta^{2}} Z \quad \text { with } \quad Z=-2 Z^{\text {wires }} \log \left(\frac{S_{21}^{D}}{S_{21}^{R}}\right)
$$

where $S_{21}^{D}$ is the transmission coefficient of the device under measurement, $S_{21}^{R}$ is the one of the reference, $Z^{\text {wires }}=400 \Omega$ is the characteristic impedance of the cable, $\Delta=2.0637 \mathrm{~mm}$ is the separation between the two wires ${ }^{1}$ and $c$ is the speed of light.

A full two-port precise calibration was used for the set-up and the error of the measurement was mainly due to the misalignments of the wires in different measurements. As an example, the figure 1 shows the absolute values and the relative error of the same measurement, in this case the transverse impedance of the ferrite frame without kicker plates, taken at two different days. In that case, the measurement was expected to give the same values where we register a relative error around $10 \%$ from 10 to $100 \mathrm{MHz}$, and at the same time the figure 1 shows that the measurement in the frequencies below $10 \mathrm{MHz}$ were very noisy. The dimensions of the plates of the model under measurement were $L=200 \mathrm{~mm}, H=65 \mathrm{~mm}$ and $P=60 \mathrm{~mm}$ (see figure 2); the dimensions of the block of ferrite were $L=150 \mathrm{~mm}, H=75 \mathrm{~mm}$ and $P=70 \mathrm{~mm}$ (see figure 3). The real dimensions of the full size kicker will be $L=368 \mathrm{~mm}, H=260 \mathrm{~mm}$ and $P=172 \mathrm{~mm}$. Therefore, it is worth noting that the magnitude of the transverse impedance from the actual measurement are different from the ones of the full size kicker, knowing that, with the same geometry, the smaller the model, the bigger the transverse impedance. As already said, our present goal is to understand the trends of the involved quantities.

\footnotetext{
${ }^{1}$ We used a standard CQ551 cable.
} 

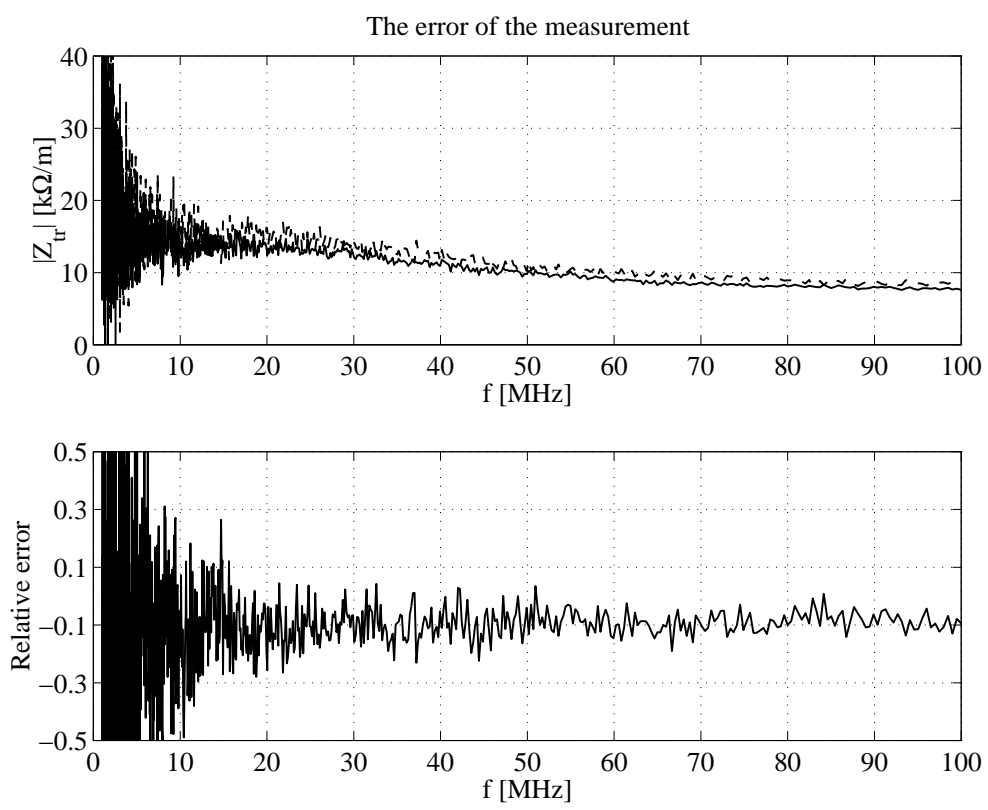

Figure 1: The error of the measurement. The plot shows the same measurement taken at two different times.

\section{Comments on the Measurement}

In the following we comment all the measurement performed on the available simplified model.

\subsection{Effect of the Termination at the PFN Port}

In the figure 4 a comparison of the transverse impedance with respect to the termination at the

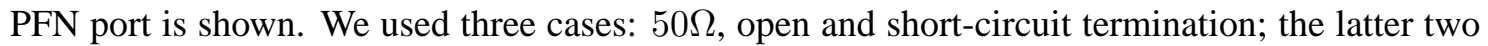
represent the extreme cases, the other terminations being included in those. Of course, opencircuit termination means that the PFN port is left as it is, which is roughly a very high impedance in parallel with a small capacitive impedance.

One can see that there is a factor 2 between the open and the short-circuit termination impedances. The short-circuit termination has the lowest transverse impedance in the whole range of measured frequencies. The $50 \Omega$ termination, which should roughly represent all the intermediate terminations, including the design $25 \Omega$ one, is always in the middle, except for the real part up to $40 \mathrm{MHz}$ where it is bigger, of around $20 \%$, than the open-circuit termination.

It is worth to note that the factor 2 is strongly dependent on the geometry and dimensions of the measured kicker. For example, previous measurement on the AGS dump kicker [4] showed roughly a factor 10 between those extreme cases. 


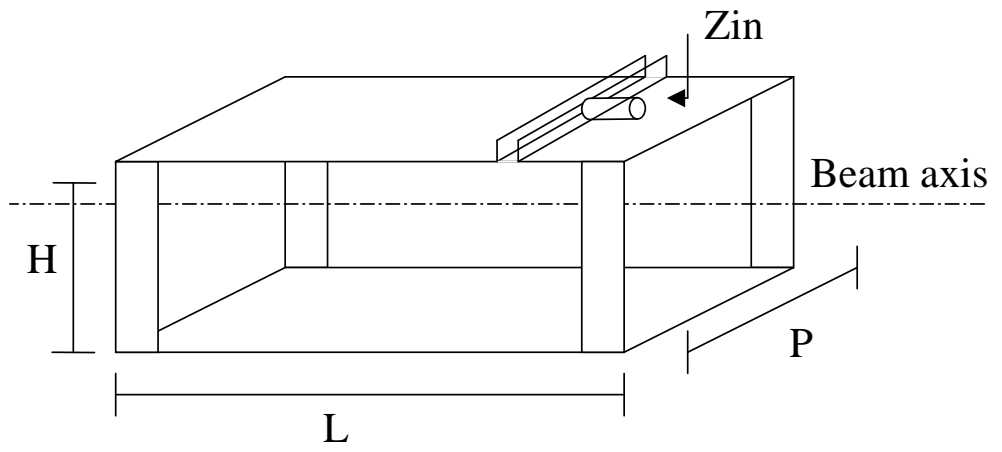

Figure 2: The geometry of the kicker plates used for the measurement. The dimensions are $L=$ 200mm, $H=65 \mathrm{~mm}$ and $P=60 \mathrm{~mm}$.

\subsection{The ferrite winding (YY Loop) and the Kicker Magnetic Field Rise Time}

In this section we discusse an intuitive approach to the YY Loop effect. Let us consider the ferrite frame shown in figure 3. It is composed by four pieces of ferrite that realize a rectangular pipe in which the particle beam is supposed to go through. Let us consider a pure dipole in the geometrical center of the transverse section; the magnetic field produced by that dipole can represent both the field produced by the kicker plates, and by the two wires we used to measure the transverse impedance. Of course, the dipole in an off-axis charged particle beam is expected to be the strongest component of the transverse kick experienced by a trailing particle.

The magnetic field penetrates the ferrite, creating a magnetic flux that goes through the ferrite arms as it is shown in figure 3. The YY loop can be seen as two coils connected in such a crossway. By using a quasi-static approach, it is apparent that a dipole flux produces two currents in opposite directions in the two coils and then the net effect is zero, that means no circulation of any current in the loop and no opposite-flux produced. Therefore, there is no effect from the YY Loop on the magnetic flux produced by a dipole. It is worth note that this statement is true for any dipole field, and therefore for both the kicker magnetic field and the dipole component of the beam. But we do not have to forget that these argument come out from a quasi-static approach.

In fact, let us consider now the input impedance of the kicker plates, namely the one seen through the PFN port, shown in the figure 5. It is clear that the input impedance is the same with and without the YY Loop up to $5 \mathrm{MHz}$, confirming the previous statements, and that for higher frequency there is a different behavior which is supposed to give different transverse impedances behaviors.

Since the magnetic field of the kicker is required to have a rise time of $200 \mathrm{~ns}$, corresponding to a highest frequency component of $5 \mathrm{MHz}$, the YY Loop does not modify the magnetic flux and the rise time. 


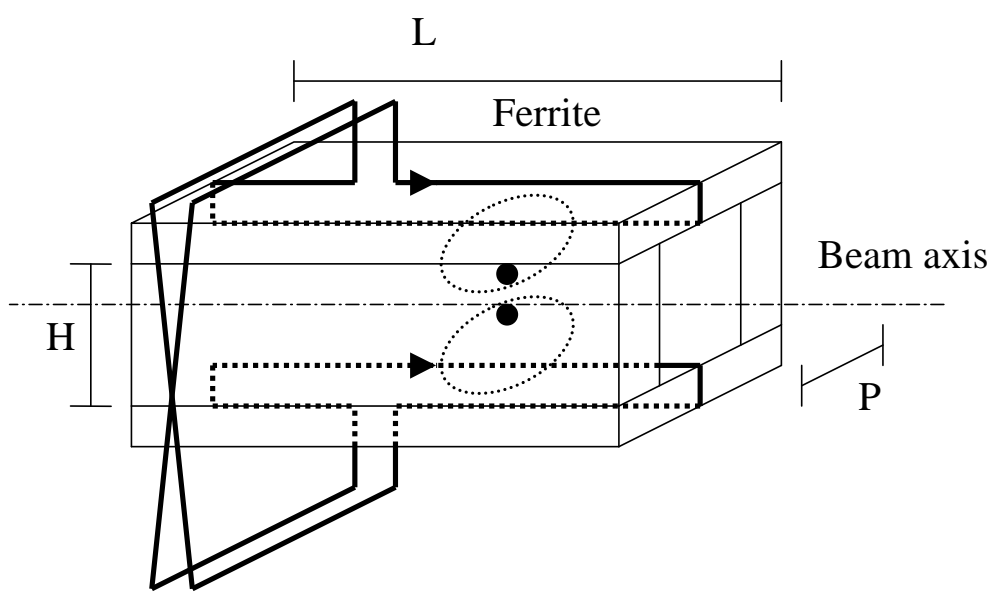

Figure 3: The geometry of the ferrite blocks. The dimensions are $L=150 \mathrm{~mm}, H=75 \mathrm{~mm}$ and $P=70 \mathrm{~mm}$. It is also shown the idea of the YY loop effect on a pure dipole magnetic field.

\subsection{The Effect of the YY loop on the Transverse Impedance}

In the figure 6 the ratio of the transverse impedances with over without the YY loop is shown. This means that numbers lower than the unity imply a reduction of the transverse impedance. For the imaginary part there is always a reduction of the transverse impedance, whereas for the real part of the open-circuit termination below $20 \mathrm{MHz}$ there is actually an increase of the impedance.

\subsection{Horizontal Wires}

In the figure 7 the transverse impedance of horizontal wires (with respect to the kicker plates) is shown. The magnitude is lower than the one of the vertical wires, but the imaginary part has the same order of magnitude. This is due to the fact that the ferrite dimension $(P=70 \mathrm{~mm})$ is smaller than in the other direction $(H=75 \mathrm{~mm})$. In any case, the real prototype will be also checked, in order to consider that impedance in the computation of the beam instability.

\section{Conclusions}

In this technical report we gave a preliminary answer on the effect of a new type of ferrite winding (YY Loop) and of the PFN termination on the transverse impedance. It is clear that the YY loop does not modify the rise time of the magnetic field produced by the kicker, having confirmation both from MAFIA simulations [7] and from the measurement described in this note. The reduction of the transverse impedance is clear at frequencies higher than $20 \mathrm{MHz}$, but the effective amount of which depends on the geometry and should be around a factor 1.5. The effect of the PFN 
port termination depends on the actual geometry. For the measured model there is a factor 2 between open and short-circuit terminations, which are the extreme cases. It is planned to verify the previous statements in the measurement of a more realistic prototype in May 2001.

\section{Acknowledgements}

We would like to thank Y.Y. Lee for fruitful discussions on his new type of winding and J. Cupolo for the technical help in the set-up of the measurement.

\section{References}

[1] S.Y. Zhang, SNS Storage Ring Impedances, BNL/SNS Technical Note n. 061, 1999.

[2] S.Y. Zhang, NSNS Transverse Instability, BNL/NSNS Technical Note n. 033, 1997.

[3] Y.Y. Lee in Accelerator Systems Advisory Committee Review, Agenda and Presentations, September $11-13,2000$.

[4] J.G. Wang and S.Y. Zhang, Measurements of Coupling Impedance for the SNS Accumulator Ring, BNL/SNS Technical Note No. 079, 2000.

[5] L.S. Walling et al., Transmission-Line Impedance Measurements for an Advanced Hadron Facility, NIM A281, pp. 433 - 447, 1989.

[6] V.G. Vaccaro, Coupling Impedance Measurement: an improved method, INFN/TC-94/023, 1994.

[7] S. Kurennoy, private communications. 

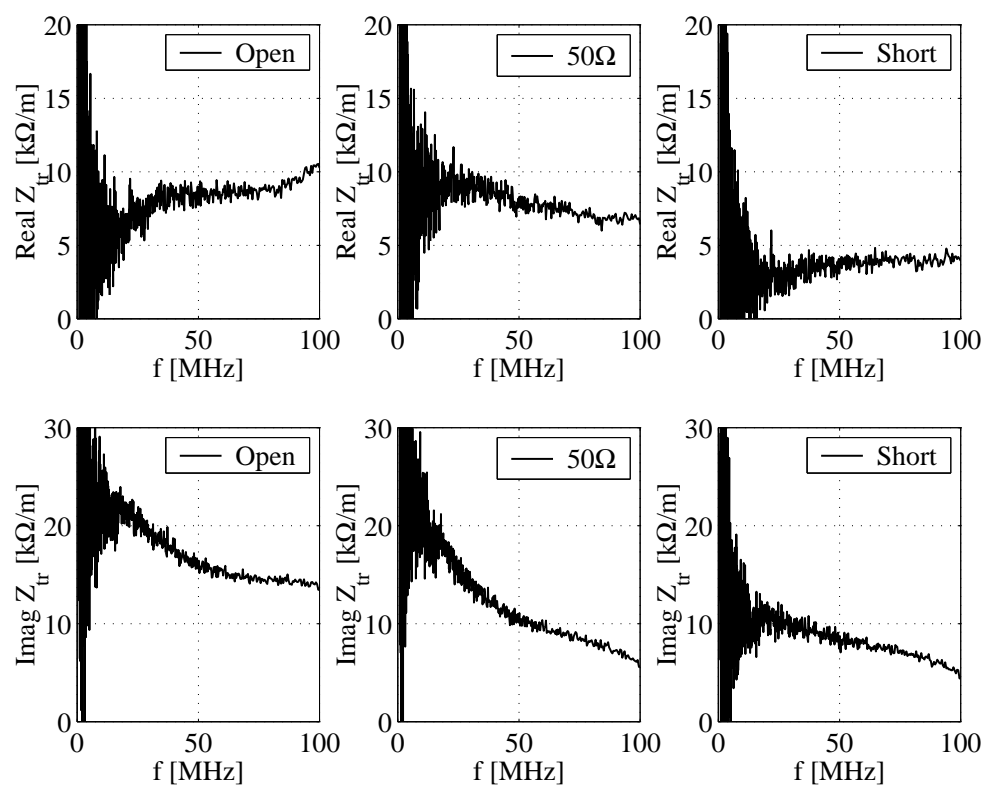

Figure 4: The transverse impedance without YY Loop with Open, $50 \Omega$ and Short-circuit termination on the PFN port.
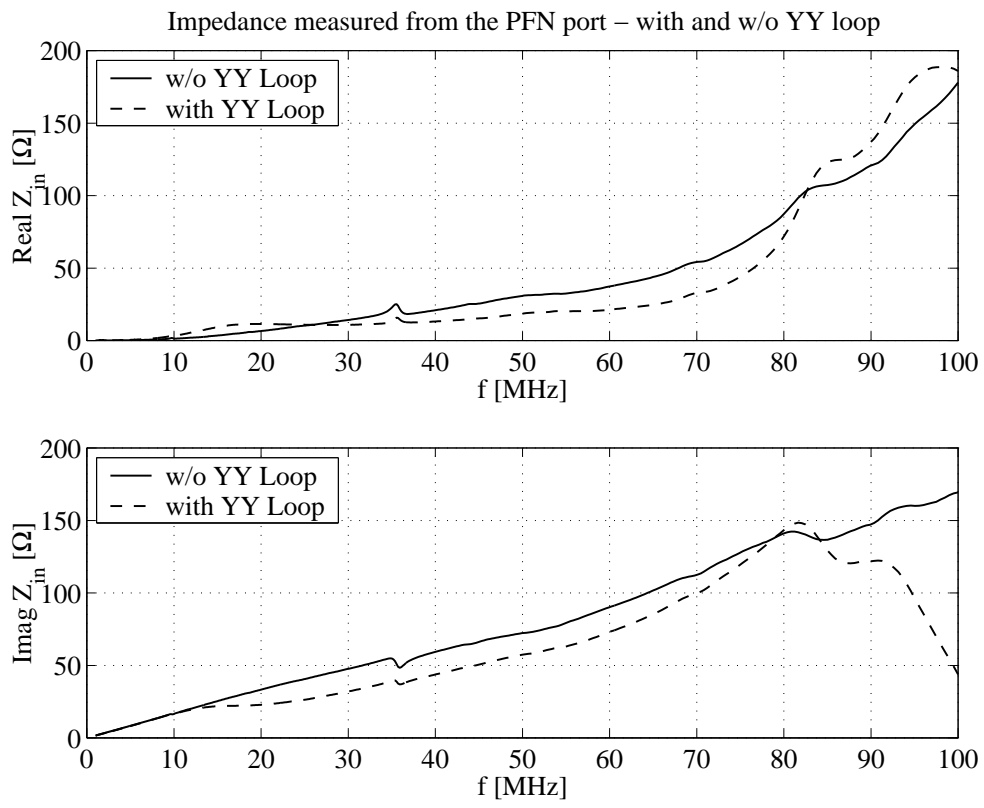

Figure 5: The input impedance measured from the PFN Port. 

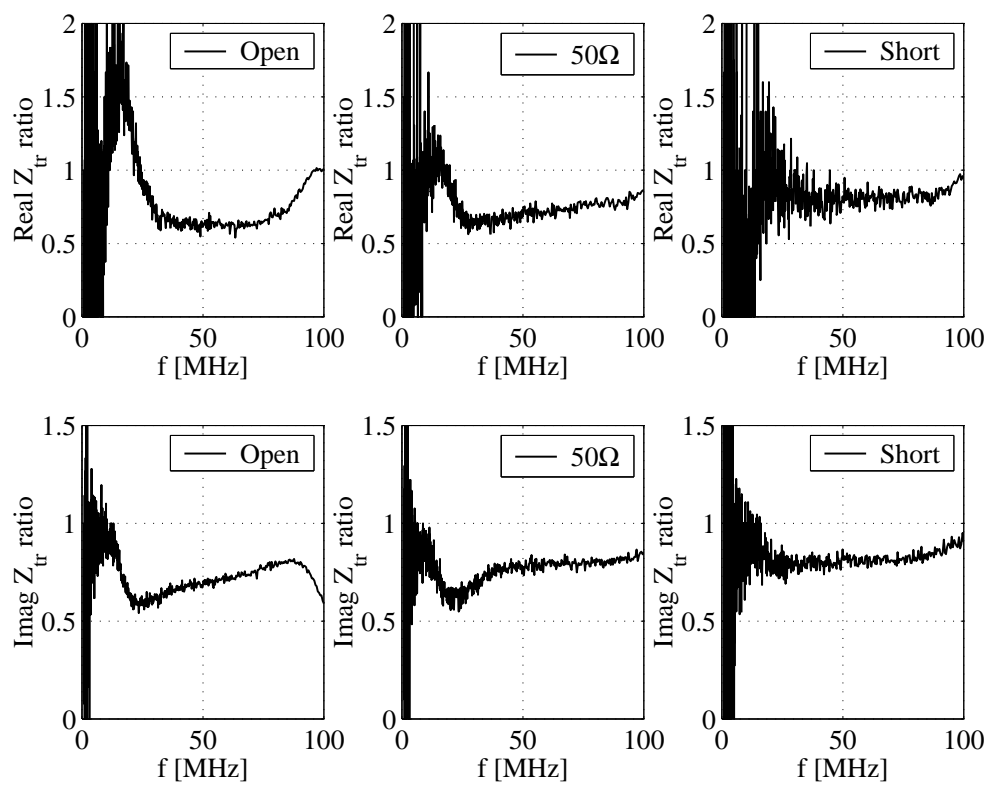

Figure 6: The transverse impedance ratio with YY Loop over without YY Loop.
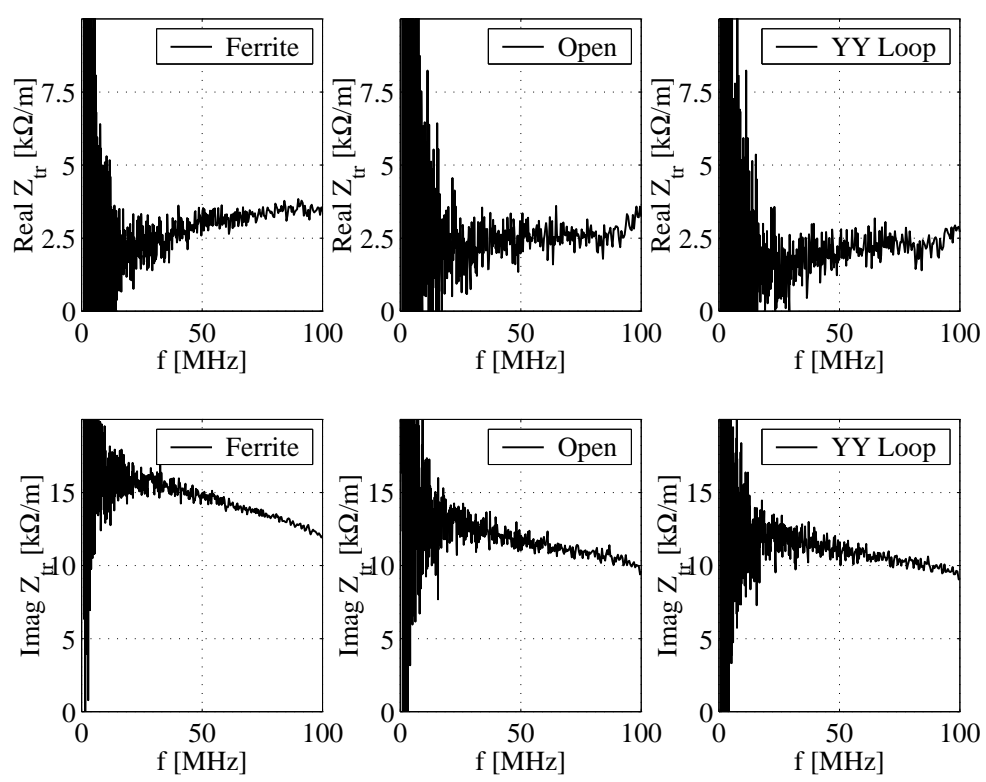

Figure 7: The transverse impedance with two horizontal wires (respect to the kicker plates). 